\title{
GEOMETRICAL FORMULATION OF THE CONFORMAL WARD IDENTITY
}

\author{
M. Kachkachi \\ Unité de Physique Mathématique, Département de Mathématiques et Informatique, \\ Faculté des Sciences et Techniques, Université Hassan $1^{\text {er }}$, \\ Settat, Morocco ${ }^{1}$ \\ and \\ The Abdus Salam International Centre for Theoretical Physics, Trieste, Italy.
}

\begin{abstract}
In this paper we use deep ideas in complex geometry that proved to be very powerful in unveiling the Polyakov measure on the moduli space of Riemann surfaces and lead to obtain the partition function of perturbative string theory for 2, 3, 4 loops. Indeed, a geometrical interpretation of the conformal Ward identity in two dimensional conformal field theory is proposed: the conformal anomaly is interpreted as a deformation of the complex structure of the basic Riemann surface. This point of view is in line with the modern trend of geometric quantizations that are based on deformations of classical structures. Then, we solve the conformal Ward identity by using this geometrical formalism.
\end{abstract}

MIRAMARE - TRIESTE

August 2002

\footnotetext{
${ }^{1}$ Permanent address.
} 


\section{Introduction}

Two-dimensional conformal fields theories on Riemann surfaces without boundary are powerful tools to deal with string theory. In particular, the dependence on the background geometry has been used to develop effective actions for the two-dimensional quantum gravity [1]. This has led to exciting developments in non-critical string theory [2] and may shed some light on the quantization programme of highest dimensional gravity. Moreover, the quantum theory of the string can be expressed in two different versions: in the canonical quantization it appears as the representation theory of Heisenberg, Virasoro, and Kac-Moody algebras. In the quantization formalism of Polyakov which is geometric and thus treats global objects, the integration over the matter field is gaussian but the integration over the zweibein is non-trivial and leads to two different settings depending on the gauge. In the conformal gauge obtained after transforming the zweibein by diffeomorphism and Weyl rescalings into a flat reference one, the functional integration analysis leads to the Liouville theory whose action yields, out of critical dimension, a measure of the violation of the conformal symmetry at the quantum level. In the light-cone gauge, which has a single non-vanishing metric mode called the Wess-Zumino field and represented geometrically by the Beltrami differential, the theory takes a local form. The resulting action is the Polyakov action which is a holomorphic functional of the Beltrami differential and is reparametrization invariant, while its variation under the Weyl rescaling produces the conformal anomaly whose strength is measured by the central charge of the model under consideration up to a conventional normalization. The form of such anomaly is universal.

\section{Classical Ward identity}

Ward identities are relations between Green's functions resulting from initial classical invariance. They are the basic means providing insight into the quantum structure of gauge theories. The use of Ward identities in Yang-Mills theory investigates the gauge dependence (this is the case of theories with composite fields). In quantum general gauge theories (both renormalized and non renormalized ones) Ward identities underly the proof of the existence of Noether charge operators with the algebraic properties required for the analysis of unitary conditions. For a two-dimensional quantum field theory, the number of the present exterior fields in the theory is the same as the number of Ward identities constraining the model. In particular, for a $2 \mathrm{~d}$ conformal model constructed on a bi-dimensional Riemann surface that is endowed with complex structures, there are two conformal Ward identities (one is the complex conjugate of the other). Moreover, exterior fields are interpreted as exterior sources of the energy-momentum tensors. This is the basic statement of the Polyakov conjecture for a two-dimensional conformal model [3]. Moreover, in the Beltrami parametrization scheme, these exterior sources are identified as Beltrami differentials $\mu$ and $\bar{\mu}$ (c.c. of $\mu$ ). Indeed, the classical conformal Ward identity 
is expressed as:

$$
\left(\delta_{\xi} \mu \frac{\delta}{\delta \mu}+\delta_{\xi} \bar{\mu} \frac{\delta}{\delta \bar{\mu}}\right) S_{C}=0
$$

where, $S_{C}$ is the classical action of an effective $2 \mathrm{~d}$ conformal model, $\xi=\xi^{z} \partial+\xi^{\bar{z}} \bar{\partial}$ is a vector field on a tangent space of a bi-dimensional Riemann surface $\Sigma ; \xi \in V e c t(\Sigma), \partial \equiv \frac{\partial}{\partial z}$ and $\delta_{\xi}$ is the diffeomorphism symmetry generator. Then, one can verify that this Ward identity encodes the conformal invariance of the classical bi-dimensional conformal model whose effective action is $S_{C}$. In a reference complex structure $(z, \bar{z})$ of this Riemann surface $\Sigma$, we express the infinitesimal diffeomorphisms transformation of the Beltrami differential as:

$$
\delta_{\xi} \mu=W_{-1} H^{z}
$$

where, $W_{-1} \equiv \bar{\partial}-\mu \partial+\mu \partial \mu$ and $H^{z} \equiv \xi^{z}+\mu_{\bar{z}}^{z} \xi^{\bar{z}}$. One can verify that equation (2) enables us to rewrite the classical conformal Ward identity as follows:

$$
W_{2} \frac{\delta S_{C}}{\delta \mu}+\overline{\mu W_{2}} \frac{\delta S_{C}}{\delta \bar{\mu}}=0 .
$$

$W_{2} \equiv \bar{\partial}-\mu \partial-2 \mu \partial \mu$ is called the Ward operator. Taking into account the holomorphic factorization of the action; $S_{C}(\mu, \bar{\mu})=S(\mu)+\bar{S}(\bar{\mu})$ with $\bar{S}(\bar{\mu})=\overline{S(\mu)}$, we get the following relation

$$
W_{2} \frac{\delta S}{\delta \mu}+\overline{\mu W_{2}} \frac{\delta \bar{S}}{\delta \bar{\mu}}=0 .
$$

Then, as $|\mu| \prec 1[4]$, it is easy to show that this equation leads to the well-known classical Ward identity:

$$
W_{2} \frac{\delta S}{\delta \mu}=0
$$

where, $\left.\Theta(z, \bar{z}) \equiv \frac{\delta S(\mu)}{\delta \mu}\right|_{\mu=\bar{\mu}=0}$ is the classical effective energy-momentum tensor of the bidimensional model under consideration. Geometrically speaking, this latter equation is interpreted as a particular case $(\mathrm{j}=2)$ of an exact $\mu$-holomorphy condition that is satisfied by a j-differential $f_{j}[5]$ :

$$
W_{j} f_{j}=0 .
$$

$W_{j} \equiv \bar{\partial}-\mu \partial-j \partial \mu$ is the generalized Ward operator whose zero modes are the $\mathrm{j}$-differentials.

\section{Quantum Ward identity}

At the quantum level, the classical action is extended to the vertex functional $\Gamma(\mu, \bar{\mu})$ which determines the Green functions of the model:

$$
\Gamma=S_{C}+\hbar \Gamma^{(1)}
$$

$\Gamma^{(1)}$ depends only on $\mu$ and $\bar{\mu}$ and is generated by correlation functions of the classical energymomentum tensor. This latter is a non-local distribution that diverges quadratically in the 
sense of the classical power counting [6]. Then, the classical Ward identity is extended to its quantum version that is satisfied by the functional $\Gamma$ modulo an inhomogeneous term of $\mu$ and $\bar{\mu}$. Such anomaly exists and is unique [7]. It is the reflection of the Weyl-Lorentz anomaly of the metric scheme modulo a local counter-term [7]. An integral form of this anomaly is given, on the complex plane, by the following:

$$
\int_{p} A(C, \mu)=\int_{p} d m \partial^{3} \mu
$$

where, $A(C, \mu)$ is a 3 -differential form in the bi-graded algebra $C_{l o c}^{*}\left(D_{0}, \Omega_{l o c}^{*}(\mu)\right)$ of local cochains defined on the connected diffeomorphism algebra $D_{0}$ and of local differential forms $\mu$-valued [8]. $C^{z}=c^{z}+\mu_{\bar{z}}^{z} c^{\bar{z}}$ is a suitable combination of the corresponding ghost fields to the diffeomorphisms parameters $\xi^{z}, \xi^{\bar{z}}$ in the BRST formalism and $d m(z)=\frac{d \bar{z} \wedge d z}{2 i}$ is the two-dimensional measure expressed in the reference complex structure $(z, \bar{z})$.

Now, the Legendre transform of the generating functional $\Gamma$ is the connected Green functional which is expressed as:

$$
Z^{c}\left[J_{\phi}, \mu, \bar{\mu}\right]=\int_{p} d m J_{\phi} \phi-\Gamma[\phi, \mu, \bar{\mu}]
$$

where, $\phi$ is a collection of fields of the model and $J_{\phi}$ are the associated exterior sources. Then, the classical Ward identity 5 is translated, at the quantum level, to the following anomalous Ward identity:

$$
W_{2} \frac{\delta Z_{v}^{c}(\mu)}{\delta \mu}=\frac{k}{12 \pi}\left(\partial^{3} \mu\right)(z)
$$

$Z_{v}^{c}[\mu, \bar{\mu}] \equiv Z^{c}\left[J_{\phi}, \mu, \bar{\mu}\right]_{\phi=0}$ and $\mathrm{k}$ is the central charge of the model. As we can verify from this latter equation, the anomaly measures the non holomorphic character of the energy-momentum tensor derived from the functional $Z_{v}^{c}$. On the other hand, the transition from the classical level to the quantum one is expressed geometrically by a quasiconformal transformation, whose dilatation coefficient is the Beltrami differential $\mu$ [4], from the reference complex structure $(z, \bar{z})$ defined by $\mu=0$ to another one $(Z, \bar{Z})$ determined by the Beltrami equation:

$$
(\bar{\partial}-\mu \partial) Z=0
$$

Then, in this geometrical setup the classical level is characterized by an exact $\mu$-holomorphy condition for a 2-differential (the classical energy-momentum tensor) however, the quantum level is characterized by a deformed one for the quantum energy-momentum tensor.

\section{Conformally covariant Ward identity}

To preserve the conformal covariance of the diffeomorphism anomaly and then to get manifest the conformal covariance of the model, a projective connection is required. This latter parametrizes a projective structure that is associated to a complex structure[4]. 


\subsection{Conformally covariant anomaly}

As a non-trivial element of the 1-cohomological space of linear applications on the diffeomorphism algebra $D_{0}$ and of local functionals in $\mu$ valued; $H_{l o c}^{1}\left(D_{0}, \Gamma_{l o c}(\mu)\right)$, the above diffeomorphism anomaly can expessed locally (up to a sign) as [8]:

$$
A(C, \mu)=\frac{-k}{24 \pi}\left(C \partial^{3} \mu-\mu \partial^{3} C\right) .
$$

Its transformation law under a conformal change of coordinates

$$
z \mapsto \omega(z)
$$

is given by:

$$
A_{\omega}=A_{z}+2 \zeta_{z}(\omega)(C \partial \mu-\mu \partial C)(z)
$$

where, $\zeta_{z}(\omega) \equiv \partial^{2} \ln \partial \omega-\frac{1}{2}(\partial \ln \partial \omega)^{2}$ is the schwarzian derivative of the function $\omega(z, \bar{z})$ with respect to the variable $z$. In particular, the schwarzian derivative of a Möbius transformation $\omega ; \omega \in S L(2, p)$ is zero and then, the anomaly 12 is conformally invariant in a projective atlas. On a general complex atlas, let us consider the following form of the anomaly:

$$
\tilde{A}(C, \mu)=\frac{k}{24 \pi}\left[C \partial^{3} \mu-\mu \partial^{3} C+2 R(C \partial \mu-\mu \partial C)\right]
$$

where, $R(z, \bar{z})$ is any complex function for the moment. However, in order to get the anomaly (15) conformally covariant and precisely a $(-1,-1)$-tensor with respect to the conformal change of coordinates (13) that is;

$$
\tilde{A}_{\omega}=(\overline{\partial \omega})^{-1}(\partial \omega)^{-1} \tilde{A}_{z}
$$

and by taking into account the following transformation laws with respect to the same conformal change of coordinates

$$
\begin{gathered}
d m(\omega)=|\partial \omega|^{2} d m(z), \\
C_{\omega}=\partial \omega C_{z} \\
\mu_{\omega}=(\overline{\partial \omega})^{-1} \partial \omega \mu_{z}
\end{gathered}
$$

one can verify that the function $R(z, \bar{z})$ should be a projective connection $[4,9]$ :

$$
W_{2} R=\partial^{3} \mu
$$

It is a deformed $\mu$-holomorphy condition for the projective connection $R$ by the diffeomorphism anomaly. Also, it is easy to show that the transformation law, with respect to the conformal change (13), of the projective connection $R$ is given by:

$$
R_{\omega}=(\partial \omega)^{-2}\left(R_{z}-\zeta_{z}(\omega)\right)
$$


Moreover, one can verify that the particular case of this projective connection is the schwarzian derivative $\zeta_{z}(\omega)$ :

$$
W_{2} \zeta_{z}(\omega)=\partial^{3} \mu
$$

Then, one can verify that the conformally covariant form of the anomaly (15) can be expressed as:

$$
\tilde{A}(C, \mu)=\frac{k}{24 \pi}\left(C L_{3}^{R}(\mu)-\mu L_{3}^{R}(C)\right)
$$

where, $L_{3}^{R} \equiv \partial^{3}+2 R \partial+\partial R$, the covariant form of the operator $\partial^{3}$, is called the third Bol's operator. Moreover, one can show (on any Riemann surface $\Sigma$ ) the relation:

$$
\int_{\Sigma} d m \mu L_{3}^{R}(C)=-\int_{\Sigma} d m C L_{3}^{R}(\mu)
$$

and hence, the integrated form of the diffeomorphism anomaly can be written as:

$$
\tilde{A}(C, \mu)=\frac{k}{12 \pi} \int_{\Sigma} d m C L_{3}^{R}(\mu) .
$$

\subsection{Conformally covariant Ward identity}

The Polyakov conjecture for a bi-dimensional conformal model states that, on the complex plane, the formal series $Z_{v}^{c}(\mu)$ is resumed by the following Wess-Zumino-Polyakov action[8]:

$$
\Gamma_{W Z P}=\frac{-k}{24 \pi} \int_{p} d m \mu \partial^{2} \ln \partial Z
$$

where, the local coordinate $Z(z, \bar{z})$ satisfies the Beltrami equation (11). Then, we have

$$
\Gamma_{W Z P}(\mu)=\frac{-k}{12 \pi} Z_{v}^{c}(\mu) .
$$

Moreover, one can verify that the functional derivation of the action (25) with respect to the Beltrami differential $\mu$ is given by:

$$
\frac{\delta \Gamma_{W Z P}}{\delta \mu}=\frac{-k}{12 \pi} \zeta_{z}(Z)
$$

Hence, by using the above equation, we get the conformally covariant form of the quantum Ward identity:

$$
\bar{\partial} T_{z z}(z, \bar{z})=L_{3}^{T}(\mu)
$$

where, $T_{z z} \equiv \frac{\delta Z_{v}^{c}}{\delta \mu}$ is the quantum energy-momentum tensor of an effective $2 \mathrm{~d}$ conformal model. This is the analogue of the deformed $\mu$-holomorphy equation that is satisfied by the schwarzian derivative given before. Then, we get its solution as:

$$
T_{z z}(z, \bar{z})=\zeta_{z}(Z)+f_{z z}(z, \bar{z})
$$

where, $f_{z z}(z, \bar{z})$ is the coefficient of a 2 -differential and then, satisfies the exact $\mu$-holomorphy equation

$$
W_{2} f_{z z}=0 .
$$


Moreover, the transformation law of the function $T$ under the holomorphic change of coordinates $z \mapsto \omega(z)$ :

$$
T_{\omega \omega}=(\partial \omega)^{2}\left(T_{z z}-\zeta_{z}(\omega)\right)
$$

shows that $T$ is not a tensor with respect to bi-dimensional conformal transformations. On the other hand, as the classical Ward identity implies that the classical energy-momentum tensor $\Theta_{z z}(z, \bar{z})$ is a 2-differential, we can re-express the quantum energy-momentum tensor in terms of the latter one as follows:

$$
T_{z z}(z, \bar{z})=\zeta_{z}(Z)+\Theta_{z z}(z, \bar{z})
$$

Then, this conformal Ward identity's solution tells us that the quantum corrections to an effective classical bi-dimensional conformal model are generated by the schwarzian derivative of a quasiconformal transformation on a Riemann surface on which the model is considered. Moreover, the transition from the classical level to the quantum one: $\Theta_{z z} \mapsto T_{z z}$ is geometrically interpreted as the passage from a reference atlas to its transform by this quasiconformal transformation.

\section{Iterative solution of the conformally covariant Ward identity}

Now, let us rewrite the local form of the conformal Ward identity as follows:

$$
\bar{\partial} T=\mu \partial T+2 T \partial \mu+\frac{k}{12 \pi} L_{3}^{R}(\mu) .
$$

Then, to determine an iterative solution of such equation on any bi-dimensional Riemann surface $\Sigma$ without boundary as a Neumann series in powers of the Beltrami differential, let us define a Cauchy-Riemann kernel $N$ on this surface by the following: for any complex valued function $f$ we have

$$
\left(\bar{\partial}^{-1} f\right)(z) \equiv \int_{\Sigma} d m(w) N(w, z) f(w) .
$$

Moreover, let us rewrite equation (33) as:

$$
\bar{\partial} \check{T}=D \bar{\partial} \check{T}+L_{3}^{R}(\mu)
$$

with, $\check{\mathrm{T}} \equiv \frac{k}{12 \pi} T$ and $\mathrm{D} \equiv(\mu \partial+2 \partial \mu) \bar{\partial}^{-1}$. As the Beltrami differential satisfies the ellipticity condition: $\mu \in C^{\infty}(\Sigma),|\mu| \prec 1$, the Cauchy integral (34) enables us to get, in the reference complex structure, the following conformally covariant Ward identity's iterative solution in powers of the Beltrami differential $\mu$ :

$$
\check{T}_{0}=\int_{\Sigma} d m_{1} N_{01} L_{3}^{R}(1)-\int_{\Sigma} d m_{1} \mu_{1} V_{01} \check{T}_{1}
$$

where, we have used the notations:

$$
i \equiv z_{i}
$$




$$
\begin{aligned}
\check{T}_{i} & \equiv \check{T}\left(z_{i}\right) \\
N_{i-1 i} & \equiv N(i-1, i) \\
V_{i-1 i} & \equiv 2 \partial_{i} N_{i-1 i}+N_{i-1 i} \partial_{i} \\
d m_{i} & \equiv \frac{d \bar{z}_{i} \wedge d z_{i}}{2 i} .
\end{aligned}
$$

Then, at any order of the perturbative series, say $(n-1)$; in another word, in any local coordinates system $\left(z_{n-1}\right)$ of the same atlas on the surface $\Sigma$ we get

$$
\check{T}_{n-1}=\int_{\Sigma} d m_{n} \mu_{n}\left(\partial_{n}^{3} N_{n-1 n}+V_{n-1 n} R_{n}\right)-\int_{\Sigma} d m_{n} V_{n-1 n} \check{T}_{n}
$$

where, $R_{n}$ is the iterative solution of the $\mu$-holomorphy equation that is satisfied by the projective connection $R$ on any Riemann surface $\Sigma$. On the complex plane, this solution was given in [4] as:

$$
R_{n}=\sum_{k=1}^{+\infty}(-1)^{-k} \int_{p} \Pi_{j=1}^{k+n}\left(d m_{j+n} \mu_{j+n}\right) \partial_{k+n}^{3} A_{k+n-1}^{p}
$$

where $A_{0}^{p} \equiv N_{01}^{p}$ is the Cauchy kernel on the complex plane and $A_{k}^{p}=\left(2 \partial_{k-1} A_{k-1}^{p}+A_{k-1}^{p} \partial_{k}\right) N_{k k+1}^{p}$. Finally, we express the quantum energy-momentum tensor as the sum of the perturbative series

$$
T_{0}=\frac{k}{12 \pi} \sum_{n=1}^{+\infty}(-1)^{n} \int_{\Sigma} \Pi_{i=1}^{n}\left(d m_{i} \mu_{i} V_{i-1 i}\right)\left(\partial_{n}^{3} V_{n-1 n}+R_{n}\right)
$$

\section{Conclusion}

We have expressed geometrically the bi-dimensional conformal Ward identity as a $\mu$-holomorphy equation on a complex Riemann surface $\Sigma$ on which the model is considered. Then, this geometrical setup enables us to get the exact solution of this conformal Ward identity as a $\mu$-holomorphic function up to the classical solution. Moreover, we have exhibited the conformal covariance character of this identity by expressing this latter in terms of conformally covariant operators like the third Bol's operator. Finally, we have developed the iterative solution of this identity as a Neumann series in powers of the Beltrami differential on any complex Riemann surface without boundary by supposing the existence of a Cauchy kernel whose explicit expression on this surface was not given. 


\section{References}

[1] N. Seiberg: Lecture at the 1990 Yukawa International Seminar on Commun Trends in Mathematics and Quantum Field Theories, Kyoto, Japan (1990)

[2] H. Verlinde, Conformal Field Theory, Two-dimensional Quantum Gravity and Quantization of Teichmuller Space, Nuc. Phys. B 337 (1990) 652

[3] A. M. Polyakov, Mod. Phys. Lett. A 2, No. 11 (1987) 893

[4] M. Kachkachi and M. Kessabi, J. Phys. A: Math. Gen. 34 (2001) 6157-6166

[5] M. Kachkachi, J. Geo. Phys. 40 (2001) 56-64

[6] L. Baulieu and M. Bellon, Phys. Lett. B196 (1987)142

[7] C. Becchi, Nucl. Phys. B 304 (1988) 513

[8] S. Lazzarini, Doctoral thesis, LAPP Annecy-Le-Vieux (1990)

[9] R. Zucchini, Commun. Math. Phys. 152 (1993) 269 